PROCEEDINGS OF THE

AMERICAN MATHEMATICAL SOCIETY

Volume 137, Number 10, October 2009, Pages 3207-3217

S 0002-9939(09)09992-4

Article electronically published on June 5, 2009

\title{
ON THE CLIQUE NUMBER OF THE GENERATING GRAPH OF A FINITE GROUP
}

\author{
ANDREA LUCCHINI AND ATTILA MARÓTI
}

(Communicated by Jonathan I. Hall)

\begin{abstract}
The generating graph $\Gamma(G)$ of a finite group $G$ is the graph defined on the elements of $G$ with an edge connecting two distinct vertices if and only if they generate $G$. The maximum size of a complete subgraph in $\Gamma(G)$ is denoted by $\omega(G)$. We prove that if $G$ is a non-cyclic finite group of Fitting height at most 2 that can be generated by 2 elements, then $\omega(G)=q+1$, where $q$ is the size of a smallest chief factor of $G$ which has more than one complement. We also show that if $S$ is a non-abelian finite simple group and $G$ is the largest direct power of $S$ that can be generated by 2 elements, then $\omega(G) \leq(1+o(1)) m(S)$, where $m(S)$ denotes the minimal index of a proper subgroup in $S$.
\end{abstract}

\section{INTRODUCTION}

The generating graph $\Gamma(G)$ of a finite group $G$ is the graph defined on the elements of $G$ with an edge connecting two distinct vertices if and only if they generate $G$. By the solution of Dixon's conjecture, it is known that $\Gamma(S)$ has "many" edges for $S$ a non-abelian finite simple group. In particular, Liebeck and Shalev 9] proved that there exists a universal positive constant $c$ such that the maximal size of a complete subgraph in $\Gamma(S)$ is at least $c \cdot m(S)$ where $m(S)$ is the minimal index of a proper subgroup in $S$. This result, in general, is best possible. Indeed, by a result of Dye [6], the group $S=S p_{2 n}(2)$ is the union of all conjugates of the maximal subgroups $O_{2 n}^{+}(2)$ and $O_{2 n}^{-}(2)$, and so $\omega(S) \leq 2^{2 n}=(2+o(1)) m(S)$.

This result of Liebeck and Shalev together with the above-mentioned remark on the symplectic group justifies the following definitions. For a finite group $G$ let the maximum size of a complete subgraph in $\Gamma(G)$ be denoted by $\omega(G)$. For a noncyclic finite group $G$ let $\sigma(G)$ denote the least number of proper subgroups of $G$ whose union is $G$. Clearly, $\omega(G) \leq \sigma(G)$. Moreover, if $\chi(G)$ denotes the chromatic number of $\Gamma(G)$ (that is, the least number of colors needed to color the vertices of $\Gamma(G)$ in such a way that the endpoints of each edge receive different colors), then we also have $\omega(G) \leq \chi(G) \leq \sigma(G)$, where the second inequality follows from the fact that $\Gamma(G)$ is $\sigma(G)$-colorable since its vertex set is the union of $\sigma(G)$ empty subgraphs.

Received by the editors July 22, 2008 .

2000 Mathematics Subject Classification. Primary 05C25, 20D10, 20 P05.

The research of the second author was supported by OTKA NK72523, OTKA T049841, NSF Grant DMS 0140578, and by a fellowship of the Mathematical Sciences Research Institute. 
The function $\sigma$ has been much investigated. For example, for a finite solvable group $G$, Tomkinson [14] showed that $\sigma(G)=q+1$, where $q$ is the minimal size of a chief factor of $G$ having more than one complement. Our first result is

Theorem 1.1. Let $G$ be a finite group with Fitting height at most 2. Then $\omega(G)=$ $\chi(G)$. Moreover, if the minimal number of generators of $G$ is 2 , then $\omega(G)=\sigma(G)$.

It is not known whether the conclusions of Theorem 1.1 are true for an arbitrary finite solvable group $G$. Blackburn [3] showed that $\omega(\operatorname{Sym}(n))=\sigma(\operatorname{Sym}(n))=2^{n-1}$ for $n$ a sufficiently large odd positive integer, and also that $\omega(\operatorname{Alt}(n))=\sigma(\operatorname{Alt}(n))=$ $2^{n-2}$ for $n$ a sufficiently large even integer not divisible by 4 . However, by [11, there are infinitely many non-abelian finite simple groups $S$ with $\omega(S)<\chi(S)<\sigma(S)$.

Still $\omega(S)$ and $\sigma(S)$ do not seem to be "far apart" for a non-abelian finite simple group $S$. In fact, Blackburn [3] asked whether $\omega(S) / \sigma(S)$ tends to 1 as the size of the non-abelian finite simple group $S$ tends to infinity. Our second result shows that there is an infinite sequence of 2-generated finite groups $G$ such that $\omega(G) / \sigma(G)$ tends to 0 as the size of $G$ tends to infinity.

Theorem 1.2. Let $S$ be a non-abelian finite simple group, let $m(S)$ be the minimal index of a proper subgroup in $S$ and let $G$ be the largest direct power of $S$ that can be generated by 2 elements. Then $\omega(G) \leq m(S)+O\left(m(S)^{14 / 15}\right)$ if $S$ is a group of Lie type and $\omega(G) \leq m(S)+O(1)$ otherwise. In particular, if $S=\operatorname{Alt}(n)$, then $\omega(G) / \sigma(G) \leq(n+O(1)) / 2^{n-2}$.

\section{Groups of Fitting height at most 2}

In this section we prove Theorem 1.1 .

Let $V$ be a finite dimensional vector space over a finite field of prime order. Let $H$ be a linear solvable group acting irreducibly and faithfully on $V$. Suppose that $H$ can be generated by 2 elements. For a positive integer $t$ we consider the semidirect product $G=V^{t} \rtimes H$, where $H$ acts in the same way on each of the $t$ direct factors. We would like to derive some information about $\omega(G)$. Put $F=\operatorname{End}_{H}(V)$.

Proposition 2.1. Assume $H=\langle x, y\rangle$ and let $\left(u_{1}, \ldots, u_{t}\right),\left(w_{1}, \ldots, w_{t}\right) \in V^{t}$. The following are equivalent:

(1) $G \neq\left\langle x\left(u_{1}, \ldots, u_{t}\right), y\left(w_{1}, \ldots, w_{t}\right)\right\rangle$;

(2) there exist $\lambda_{1}, \ldots, \lambda_{t} \in F$ and $w \in V$ with $\left(\lambda_{1}, \ldots, \lambda_{t}, w\right) \neq(0, \ldots, 0,0)$ such that $\sum_{i} \lambda_{i} u_{i}=w-w x$ and $\sum_{i} \lambda_{i} w_{i}=w-w y$.

Proof. Let $a=x\left(u_{1}, \ldots, u_{t}\right), b=y\left(w_{1}, \ldots, w_{t}\right), K=\langle a, b\rangle$. First we prove, by induction on $t$, that if $K \neq G$, then (2) holds. Let $\bar{a}=x\left(u_{1}, \ldots, u_{t-1}, 0\right)$, $\bar{b}=y\left(w_{1}, \ldots, w_{t-1}, 0\right), \bar{K}=\langle\bar{a}, \bar{b}\rangle$. If $\bar{K} ¥ V^{t-1} H$, then, by induction, there exist $\lambda_{1}, \ldots, \lambda_{t-1} \in F$ and $w \in V$ with $\left(\lambda_{1}, \ldots, \lambda_{t-1}, w\right) \neq(0, \ldots, 0,0)$ such that $\sum_{i} \lambda_{i} u_{i}=w-w x$ and $\sum_{i} \lambda_{i} w_{i}=w-w y$. In this case $\lambda_{1}, \ldots, \lambda_{t-1}, 0$ and $w$ are the requested elements. So we may assume $\bar{K} \cong V^{t-1} H$. Set $V_{t}=$ $\{(0, \ldots, 0, v) \mid v \in V\}$. We have $\bar{K} V_{t}=K V_{t}=G$ and $K \neq G$; this implies that $K$ is a complement of $V_{t}$ in $G$ and therefore there exists $\delta \in \operatorname{Der}\left(\bar{K}, V_{t}\right)$ such that $\delta(\bar{a})=u_{t}$ and $\delta(\bar{b})=w_{t}$. However, by Propositions 2.7 and 2.10 of 2], we have $H^{1}\left(\bar{K}, V_{t}\right) \cong F^{t-1}$. More precisely if $\delta \in \operatorname{Der}\left(\bar{K}, V_{t}\right)$, then there exist an inner derivation $\delta_{w} \in \operatorname{Der}(H, V)$ and $\lambda_{1}, \ldots, \lambda_{t-1} \in F$ such that for each 
$g\left(v_{1}, \ldots, v_{t-1}, 0\right) \in \bar{K}$ we have $\delta\left(g\left(v_{1}, \ldots, v_{t-1}, 0\right)\right)=\delta_{w}(g)+\lambda_{1} v_{1}+\cdots+\lambda_{t-1} v_{t-1}=$ $w g-w+\lambda_{1} v_{1}+\cdots+\lambda_{t-1} v_{t-1}$. In particular $u_{t}=w x-w+\lambda_{1} u_{1}+\cdots+\lambda_{t-1} u_{t-1}$ and $w_{t}=w x-w+\lambda_{1} w_{1}+\cdots+\lambda_{t-1} w_{t-1}$.

Conversely, if (2) holds, then $\left\langle h\left(v_{1}, \ldots, v_{t}\right) \mid w-w h=\lambda_{1} v_{1}+\cdots+\lambda_{t} v_{t}\right\rangle$ is a proper subgroup of $G$ containing $K$.

Let $n$ be the dimension of $V$ over $F$. We may identify $H=\langle x, y\rangle$ with a subgroup of $G L(n, F)$. In this identification $x$ and $y$ become two $n \times n$ matrices $X$ and $Y$ with coefficients in $F$. Let $\left(u_{1}, \ldots, u_{t}\right),\left(w_{1}, \ldots, w_{t}\right) \in V^{t}$. Then every $u_{i}$ and $w_{j}$ can be viewed as a $1 \times n$ matrix. Denote the $t \times n$ matrix with rows $u_{1}, \ldots, u_{t}$ (resp. $w_{1}, \ldots, w_{t}$ ) by $A$ (resp. B). By Proposition 2.1, the elements $x\left(u_{1}, \ldots, u_{t}\right)$, $y\left(w_{1}, \ldots, w_{t}\right)$ generate a proper subgroup of $G$ if and only if there exists a non-zero vector $\left(\lambda_{1}, \ldots, \lambda_{t} ; \mu_{1}, \ldots \mu_{n}\right)$ in $F^{t+n}$ such that

$$
\left\{\begin{array}{l}
\left(\lambda_{1}, \ldots, \lambda_{t}\right) A=\left(\mu_{1}, \ldots, \mu_{n}\right)(1-X) \\
\left(\lambda_{1}, \ldots, \lambda_{t}\right) B=\left(\mu_{1}, \ldots, \mu_{n}\right)(1-Y)
\end{array} .\right.
$$

This is equivalent to saying that there exist elements $\bar{X}$ and $\bar{Y}$ in $G$ such that $\langle\bar{X}, \bar{Y}\rangle=G$ with the property that $\bar{X}$ maps to $x$ and $\bar{Y}$ maps to $y$ under the projection from $G$ to $H$ if and only if there exist $t \times n$ matrices $A$ and $B$ with

$$
\operatorname{rank}\left(\begin{array}{cc}
1-X & 1-Y \\
A & B
\end{array}\right)=n+t .
$$

From this it immediately follows that $G$ cannot be generated by 2 elements if $t>n$ (hence $\omega(G)=1$ in this case). Notice also that if $X$ and $Y$ are two $n \times n$ matrices generating the matrix group $H$, then the linear map $\alpha: F^{n} \rightarrow F^{n} \times F^{n}, w \mapsto$ $(w(1-X), w(1-Y))$ is injective (if $w \in \operatorname{ker} \alpha$, then $w X=w Y=w$ against the fact that $X$ and $Y$ generate a non-trivial irreducible group); the matrix $\left(\begin{array}{lll}1-X & 1-Y\end{array}\right)$ has rank $n$, and so it is possible to find $A$ and $B$ satisfying (11) whenever $t \leq n$. Hence $3 \leq \omega\left(V^{n} \rtimes H\right) \leq \omega(G)$ whenever $t \leq n$. The case $t=n$ is of special importance. In this case our observations yield

Proposition 2.2. Let $t=n$. Assume that $X_{1}, \ldots, X_{\omega}$ pairwise generate $H$. Then there exist elements $\bar{X}_{1}, \ldots, \bar{X}_{\omega}$ pairwise generating $G$ (so that for all $i$ with $1 \leq$ $i \leq \omega$ the element $X_{i}$ is the projection of $\bar{X}_{i}$ under the projection from $G$ to $H$ ) if and only if there exist $n \times n$ matrices $A_{1}, \ldots, A_{\omega}$ such that for all $i$ and $j$ with $1 \leq i<j \leq \omega$ we have

$$
\operatorname{det}\left(\begin{array}{cc}
1-X_{i} & 1-X_{j} \\
A_{i} & A_{j}
\end{array}\right) \neq 0
$$

From now on let $H$ be a nilpotent finite group that can be generated by 2 elements with an irreducible (but not necessarily faithful) action $\rho: H \rightarrow G L(V)$. Let $F=\operatorname{End}_{H}(V)$ and let $n=\operatorname{dim}_{F}(V)$. The Sylow subgroups of $H$ are either cyclic or non-cyclic and 2-generated. Let $\pi_{1}$ be the set consisting of those prime divisors of $|H|$ whose corresponding Sylow subgroups are not cyclic, and let $\pi_{2}$ be the set of all other prime divisors of $|H|$. Let $p$ be the smallest prime in $\pi_{1}$. (If $\pi_{1}=\emptyset$, then set $p=\infty$.) We can find two generators $x$ and $y$ of $H$ such that $|x|$ is divisible only by primes in $\pi_{1}$ (if $\pi_{1}=\emptyset$ we take $x=1$ ). Let $X=x^{\rho}, Y=y^{\rho}$, and $u=\min (p,|V|)$. Clearly $\sigma\left(V^{t} \rtimes H^{\rho}\right) \leq u+1$.

Proposition 2.3. With the notation and assumptions above we have $\omega\left(V^{t} \rtimes H^{\rho}\right)=$ $u+1$ if $t \leq n$ and $\omega\left(V^{t} \rtimes H^{\rho}\right)=1$ otherwise. 
Proof. By our observations above, to prove Proposition 2.3, it is sufficient to show that $u+1 \leq \omega\left(V^{n} \rtimes H^{\rho}\right)$. To see this it is sufficient to verify that there exist $A, B_{0}, \ldots, B_{u-1} \in V^{n}$ such that the elements $X, Y B_{0}, X Y B_{1}, X^{2} Y B_{2}, \ldots$, $X^{u-1} Y B_{u-1}$ pairwise generate $V^{n} \rtimes H^{\rho}$.

We need to consider two different cases.

Case 1: $X \neq 1$.

Notice that $Z\left(H^{\rho}\right) \leq\left(\operatorname{End}_{H}(V)\right)^{*}$; hence $Z\left(H^{\rho}\right)$ is a subgroup of $F^{*}$. This implies

- $|X|$ divides $|F|-1$ (in particular $p \leq|F|-1$ );

- for any $h \in H, V$ is a completely reducible $\langle h\rangle$-module (indeed any prime divisor of $\left|H^{\rho}\right|$ divides $\left|Z\left(H^{\rho}\right)\right|$, and hence it is coprime with $\left.|F|\right)$.

The second remark implies that we may write $x$ in the form

$$
X=\left(\begin{array}{ll}
1 & 0 \\
0 & C
\end{array}\right),
$$

where 1 denotes the identity $\ell \times \ell$ matrix for some non-negative integer $\ell$ with $\ell<n$ and $C$ is an invertible $(n-\ell) \times(n-\ell)$ matrix which does not admit 1 as an eigenvalue. Decompose $Y$ and $1-Y$ as block matrices in the following way:

$$
Y=\left(\begin{array}{l}
Y_{1} \\
Y_{2}
\end{array}\right)
$$

and

$$
1-Y=\left(\begin{array}{c}
T_{1}-Y_{1} \\
T_{2}-Y_{2}
\end{array}\right)
$$

where $Y_{1}$ and $T_{1}-Y_{1}$ denote the matrices consisting of the first $\ell$ rows of $Y$ and $1-Y$ respectively and $Y_{2}$ and $T_{2}-Y_{2}$ denote the matrices consisting of the remaining rows of $Y$ and $1-Y$ respectively. Since

$$
\operatorname{rank}(1-X \quad 1-Y)=\operatorname{rank}\left(\begin{array}{cc}
0 & T_{1}-Y_{1} \\
1-C & T_{2}-Y_{2}
\end{array}\right)=n
$$

we deduce that $\operatorname{rank}\left(T_{1}-Y_{1}\right)=\ell$. Let $D$ be an $(n-\ell) \times n$ matrix such that

$$
\operatorname{det}\left(\begin{array}{c}
T_{1}-Y_{1} \\
D
\end{array}\right) \neq 0
$$

By Theorem 2.2, we look for $A, B_{0}, \ldots, B_{p-1}$ such that

$$
\operatorname{det}\left(\begin{array}{cc}
1-X & 1-X^{r} Y \\
A & B_{r}
\end{array}\right) \neq 0 \text { and } \operatorname{det}\left(\begin{array}{cc}
1-X^{r} Y & 1-X^{s} Y \\
B_{r} & B_{s}
\end{array}\right) \neq 0
$$

for all $r$ and $s$ such that $0 \leq r \leq s \leq p-1$. Since $p$ divides $|F|-1$, there exist $p$ pairwise distinct elements $b_{0}, \ldots, b_{p-1} \in F^{*}$. Consider the following $p \times p$ matrices:

$$
A=\left(\begin{array}{ll}
1 & 0 \\
0 & 0
\end{array}\right), \quad B_{i}=\left(\begin{array}{c}
b_{i} Y_{1} \\
D
\end{array}\right)
$$

for all $i$ with $0 \leq i \leq p-1$, where 1 in the definition of $A$ denotes the $\ell \times \ell$ identity matrix. We prove that $A, B_{0}, \ldots, B_{p-1}$ are the matrices we are looking for. Notice that

$$
X^{r} Y=\left(\begin{array}{c}
Y_{1} \\
C^{r} Y_{2}
\end{array}\right) ;
$$


hence

$$
\begin{aligned}
\operatorname{det}\left(\begin{array}{cc}
1-X & 1-X^{r} Y \\
A & B_{r}
\end{array}\right) & =\operatorname{det}\left(\begin{array}{ccc}
0 & 0 & T_{1}-Y_{1} \\
0 & 1-C & * \\
1 & 0 & * \\
0 & 0 & D
\end{array}\right) \\
& = \pm \operatorname{det}(1-C) \operatorname{det}\left(\begin{array}{c}
T_{1}-Y_{1} \\
D
\end{array}\right) \neq 0
\end{aligned}
$$

On the other hand, if $r \neq s$, then

$$
\begin{gathered}
\operatorname{det}\left(\begin{array}{cc}
1-X^{r} Y & 1-X^{s} Y \\
B_{r} & B_{s}
\end{array}\right)=\operatorname{det}\left(\begin{array}{cc}
1-X^{r} Y & X^{r} Y-X^{s} Y \\
B_{r} & B_{s}-B_{r}
\end{array}\right) \\
=\operatorname{det}\left(\begin{array}{cc}
T_{1}-Y_{1} & 0 \\
* & \left(C^{r}-C^{s}\right) Y_{2} \\
b_{r} Y_{1} & \left(b_{s}-b_{r}\right) Y_{1} \\
D & 0
\end{array}\right)=\operatorname{det}\left(\begin{array}{c}
T_{1}-Y_{1} \\
D
\end{array}\right) \operatorname{det}\left(\begin{array}{c}
\left(C^{r}-C^{s}\right) Y_{2} \\
\left(b_{s}-b_{r}\right) Y_{1}
\end{array}\right) \\
=\left(b_{s}-b_{r}\right) \operatorname{det}\left(C^{r}-C^{s}\right) \operatorname{det}\left(\begin{array}{c}
T_{1}-Y_{1} \\
D
\end{array}\right) \operatorname{det}\left(\begin{array}{c}
Y_{2} \\
Y_{1}
\end{array}\right),
\end{gathered}
$$

which is non-zero if and only if $\operatorname{det}\left(C^{r}-C^{s}\right)=\operatorname{det}\left(C^{r}\left(1-C^{s-r}\right)\right) \neq 0$. To show that the matrix $1-C^{s-r}$ is non-singular it is sufficient to see that 1 is not an eigenvalue of $C^{s-r}$. Since $V$ is a completely reducible $F\langle X\rangle$-module, $C$ can be diagonalised over a suitable field extension of $F$. Let $\beta$ be an arbitrary eigenvalue of $C^{s-r}$. Then $\beta=\gamma^{s-r}$ for some eigenvalue $\gamma$ of $C$. Now $\gamma$ is different from 1 by our choice of $C$. Finally since $0<s-r<p$ and since no prime smaller than $p$ divides $|X|$ we see that $\gamma^{s-r}$ cannot be 1 . This settles Case 1 .

Case 2: $X=1$.

In this case $H^{\rho}=\langle Y\rangle$ is a cyclic group and $V$ is an absolutely irreducible $F H$ module. Hence $V=F$ and $n=1$. We have $u \leq|F|$ and if $0 \neq A \in V$ and $B_{0}, B_{1}, \ldots, B_{u-1}$ are distinct elements of $V$, then, by Proposition 2.1. $A, Y B_{0}$, $Y B_{1}, \ldots, Y B_{u-1}$ pairwise generate $V \rtimes H^{\rho}$. This proves Proposition 2.3.

Let $G$ be a finite solvable group, and let $\mathcal{A}$ be a set of representatives for the irreducible $G$-groups that are $G$-isomorphic to a complemented chief factor of $G$. For $A \in \mathcal{A}$, let $R_{G}(A)$ be the smallest normal subgroup contained in $C_{G}(A)$ with the property that $C_{G}(A) / R_{G}(A)$ is $G$-isomorphic to a direct product of copies of $A$ and it has a complement in $G / R_{G}(A)$. The factor group $C_{G}(A) / R_{G}(A)$ is called the $A$ crown of $G$. The non-negative integer $\delta_{G}(A)$ defined by $C_{G}(A) / R_{G}(A) \cong_{G} A^{\delta_{G}}(A)$ is called the $A$-rank of $G$ and it coincides with the number of complemented factors in any chief series of $G$ that are $G$-isomorphic to $A$. If $\delta_{G}(A) \neq 0$, then the $A$-crown is the socle of $G / R_{G}(A)$. The notion of crown was introduced by Gaschütz in [7.

Proposition 2.4. Let $G$ and $\mathcal{A}$ be as above. Let $x_{1}, \ldots, x_{u}$ be elements of $G$ such that $\left\langle x_{1}, \ldots, x_{u}, R_{G}(A)\right\rangle=G$ for any $A \in \mathcal{A}$. Then $\left\langle x_{1}, \ldots, x_{u}\right\rangle=G$.

Proof. Let $H=\left\langle x_{1}, \ldots, x_{u}\right\rangle$ and suppose that $H R_{G}(A)=G$ for any $A \in \mathcal{A}$. There exists a normal subgroup $N$ of $G$ of minimum order with respect to the property $H N=G$. Assume by contradiction that $N \neq 1$ and choose $M$ such that $A=N / M$ is a chief factor of $G$. Since $H M \neq G$, we have that $A$ is a complemented chief factor and $(H M / M)\left(R_{G}(A) M / M\right)=G / M=(H M / M)(N / M)$. By Proposition 11 
of [5] and the fact that $R_{G / M}(A)=R_{G}(A) M / M$, we deduce $H M=G$, against the choice of $N$.

Let $d(X)$ denote the minimal number of generators of the finite group $X$.

Proposition 2.5. Let $G$ be a finite group of Fitting height equal to 2 . If $d(G)=2$, then $\omega(G)=\sigma(G)$.

Proof. Clearly we may assume that the Frattini subgroup Frat $(G)$ of $G$ is trivial. Then the Fitting subgroup Fit $(G)$ coincides with the direct product of the minimal normal subgroups of $G$ (see [13, 5.2.15]). Let $V$ be the subgroup of $\operatorname{Fit}(G)$ generated by the non-central minimal normal subgroups of $G$. Now $V$ is an abelian normal subgroup of a finite group with trivial Frattini subgroup, so $V$ is complemented in $G$ (see [13, 5.2.13]). So we have that $G=V \rtimes H$ for some nilpotent group $H$ with $d(H) \leq 2$. Let $\mathcal{Z}$ be the set of $G$-irreducible modules $G$-isomorphic to some factor of $V$. We have that $V=\prod_{M \in \mathcal{Z}} V_{M}$, where $V_{M}$ is the product of the minimal normal subgroups $G$-isomorphic to $M$. If $M \in \mathcal{Z}$ and $\rho_{M}: H \rightarrow G L(M)$ is the action of $H$ on $M$, then $R_{G}(M)=C_{H}(M) \times \prod_{L \in \mathcal{Z}, L \neq M} V_{L}$ and $G / R_{G}(M) \cong V_{M} \rtimes H^{\rho_{M}}=$ $M^{t_{M}} \rtimes H^{\rho_{M}}$ for some positive integer $t_{M}$. Notice that $d\left(M^{t_{M}} \rtimes H^{\rho_{M}}\right) \leq 2$ for all $M \in \mathcal{Z}$.

For the finite nilpotent group $H$ choose $p, x$, and $y$ as in the preceding paragraph of the statement of Proposition 2.3. Put $\tau=\min _{M \in \mathcal{Z}}\{|M|\}$. (Note that $\mathcal{Z} \neq \emptyset$, for otherwise $V=1$ and $G=H$ is nilpotent.) Then $\sigma(G)=1+\min \{\tau, p\}$. Put $\sigma=\sigma(G)$. By Proposition 2.3, for any $M \in \mathcal{Z}$ there exist $A_{M}, B_{0, M}, \ldots, B_{\sigma-2, M}$ such that the $\sigma$ elements

$$
x^{\rho_{M}} A_{M}, y^{\rho_{M}} B_{0, M}, \ldots,\left(x^{\sigma-2} y\right)^{\rho_{M}} B_{\sigma-2, M}
$$

pairwise generate $M^{t_{M}} \rtimes H^{\rho_{M}}$. Put

$$
a=\prod_{M \in \mathcal{Z}} A_{M}, \quad \text { and } \quad b_{i}=\prod_{M \in \mathcal{Z}} B_{i, M}
$$

for all $i$ such that $0 \leq i \leq \sigma-2$. Finally consider the set

$$
\Omega=\left\{x a, y b_{0}, x y b_{1}, \ldots, x^{\sigma-2} y b_{\sigma-2}\right\} .
$$

We claim that two distinct elements $\omega_{1}, \omega_{2}$ of $\Omega$ generate $G$. Indeed, take $M \in$ $\mathcal{A}$. If $G$ centralizes $M$, then $V \leq R_{G}(M)$; otherwise $M \in \mathcal{Z}$. In both cases $\left\langle\omega_{1}, \omega_{2}, R_{G}(M)\right\rangle=G$; hence, by Proposition 2.4, we have $\left\langle\omega_{1}, \omega_{2}\right\rangle=G$. This proves Proposition 2.5.

We are now in the position to prove Theorem 1.1

Let $G$ be as in the statement of Theorem 1.1. If $d(G)>2$, then $\Gamma(G)$ is the empty graph and so $\omega(G)=\chi(G)=1$. So assume that $d(G) \leq 2$. If the Frattini subgroup of $G$ is denoted by $\operatorname{Frat}(G)$, then $\omega(G)=\omega(G / \operatorname{Frat}(G))$ and $\chi(G)=\chi(G / \operatorname{Frat}(G))$. Moreover, if $G$ is non-cyclic, then $\sigma(G)=\sigma(G / \operatorname{Frat}(G))$. Hence we may assume that $\operatorname{Frat}(G)=1$.

Let $G$ be cyclic. Since Frat $(G)=1$, the cyclic group $G$ is the direct product of say $t$ cyclic groups of distinct prime orders. Let $S$ be the set of generators of $G$. In the graph $\Gamma(G)$ every vertex in $S$ is connected to every other vertex in $\Gamma(G)$. Thus, if $\Gamma(G) \backslash S$ denotes the graph obtained from $\Gamma(G)$ by removing all vertices from $S$ together with all edges having an endpoint in $S$, then $\omega(G)$ equals the maximum size of a complete subgraph in the graph $\Gamma(G) \backslash S$ plus $|S|$, and $\chi(G)$ equals the 
chromatic number of the graph $\Gamma(G) \backslash S$ plus $|S|$. Now $G$ has $t$ maximal subgroups each of which is cyclic. We may choose a generator from each of these maximal subgroups. Since any distinct pair of these elements generate $G$, we have a complete subgraph of size $t$ in the graph $\Gamma(G) \backslash S$. On the other hand, the graph $\Gamma(G) \backslash S$ can be expressed as the union of $t$ empty subgraphs (coming from the $t$ maximal subgroups of $G$ ); hence it is $t$-colorable and so the chromatic number of $\Gamma(G) \backslash S$ is at most $t$. These observations yield $t+|S| \leq \omega(G) \leq \chi(G) \leq t+|S|$; hence $\omega(G)=\chi(G)$.

We may now also assume that $d(G)=2$. Also, by Proposition 2.5] we assume that $G$ is nilpotent. Then, since $\operatorname{Frat}(G)=1$, we have $G=C \times N_{p_{1}} \times \ldots \times N_{p_{t}}$ for some positive integer $t$, where $p_{1}<\ldots<p_{t}$ are distinct primes, $N_{p_{j}}=C_{p_{j}} \times C_{p_{j}}$ for all $j$ with $1 \leq j \leq t$, and $C$ is a cyclic group that is a direct product of cyclic groups of prime orders different from $p_{j}$ for $j$ with $1 \leq j \leq t$. Let $N$ be the normal subgroup of $G$ for which $G / N \cong N_{p_{1}}=C_{p_{1}} \times C_{p_{1}}$. Then $\sigma(G) \leq \sigma(G / N) \leq p_{1}+1$. For each $j$ with $1 \leq j \leq t$ let $a_{1, j}, a_{2, j}, \ldots, a_{p_{1}+1, j}$ be non-identity elements from $N_{p_{j}}$ generating distinct cyclic subgroups in $N_{p_{j}}$. Let $c$ be a generator from $C$. For any $i$ with $1 \leq i \leq p_{1}+1$ let $a_{i}$ be the element $\left(c, a_{i, 1}, \ldots, a_{i, t}\right)$ from $G$. Clearly, $\left\{a_{1}, \ldots, a_{p_{1}+1}\right\}$ spans a complete subgraph in $\Gamma(G)$. Hence $p_{1}+1 \leq \omega(G) \leq \sigma(G) \leq$ $p_{1}+1$, that is, $\omega(G)=\sigma(G)$.

\section{DiRECT PRODUCTS OF NON-ABELIAN SIMPLE GROUPS}

In this section we prove Theorem 1.2 .

Our first result (Proposition 3.1) was also proved (independently) by Abdollahi and Jafarian Amiri in [1].

Proposition 3.1. Let $S$ be a non-abelian finite simple group. Then for any positive integer $n$ we have $\sigma\left(S^{n}\right)=\sigma(S)$, where $S^{n}$ denotes the direct product of $n$ copies of $S$.

Proof. The inequality $\sigma\left(S^{n}\right) \leq \sigma(S)$ follows at once from the observation that if $\left\{M_{i}\right\}$ is a set of proper subgroups of $S$ with $S=\bigcup_{i} M_{i}$, then $\left\{M_{i} \times S^{n-1}\right\}$ is a set of proper subgroups of $S^{n}$ with $S^{n}=\bigcup_{i}\left(M_{i} \times S^{n-1}\right)$.

Let $\left\{Y_{1}, \ldots, Y_{\tau}\right\}$ be a set of proper subgroups of $S^{n}$ such that $S^{n}=\bigcup_{i=1}^{\tau} Y_{i}$. Suppose also that $\tau$ is as small as possible (that is, $\tau=\sigma\left(S^{n}\right)$ ). Put $\sigma=\sigma(S)$. We need to show that $\sigma \leq \tau$.

We may assume that the $Y_{i}$ 's are maximal subgroups of $S^{n}$. What are the maximal subgroups of $S^{n}$ ? They are of the following two kinds:

- product type: $P_{M, i}=\left\{\left(x_{1}, \ldots, x_{n}\right) \in S^{n} \mid x_{i} \in M\right\}$, where $M$ is a maximal subgroup of $S$;

- diagonal type: $D_{i, j, \phi}=\left\{\left(x_{1}, \ldots, x_{n}\right) \mid x_{j}=x_{i}^{\phi}\right\}$, where $\phi \in \operatorname{Aut}(S)$.

Without loss of generality assume that $Y_{i}$ is of product type if $i \leq a$ and $Y_{i}$ is of diagonal type if $a<i \leq \tau$ for some non-negative integer $a$ at most $\tau$. We may assume that $a<\sigma$ for otherwise $\sigma \leq a \leq \tau$ in which case we are done.

Let $I$ be the set of those indices $i$ with $1 \leq i \leq n$ for which there exists a maximal subgroup $M$ of $S$ and an index $j$ with $1 \leq j \leq a$ such that $Y_{j}=P_{M, i}$. For every $i \in I$ let $\mathcal{M}_{i}$ be the set of those maximal subgroups $M$ of $S$ for which there exists an index $j$ with $1 \leq j \leq a$ such that $Y_{j}=P_{M, i}$. Define $\Omega_{i}=S \backslash\left(\bigcup_{M \in \mathcal{M}_{i}} M\right)$. Note that $\Omega_{i}$ has cardinality at least $\sigma-a$. Now for each index $j$ with $1 \leq j \leq n$, let $\Delta_{j}$ be a subset of $S$ of cardinality $\sigma-a$ with the property that $\Delta_{i} \subseteq \Omega_{i}$ whenever 
$i \in I$. Consider the subset $\Gamma=\prod_{j=1}^{n} \Delta_{j}$ of $S^{n}$. Clearly $|\Gamma|=(\sigma-a)^{n}$. Since $\Gamma \cap\left(\bigcup_{j \leq a} Y_{j}\right)=\emptyset$, we must have $\Gamma \subseteq \bigcup_{j>a} Y_{j}$. Notice that $|M \cap \Gamma| \leq(\sigma-a)^{n-1}$ for any maximal subgroup of $S^{n}$ of diagonal type. This means that $\Gamma$ is a subset of no less than $\sigma-a$ maximal subgroups of diagonal type. Hence $\sigma-a \leq \tau-a$, which is exactly what we wanted.

Let $S$ be a non-abelian finite simple group. Define $\delta=\delta(S)$ to be the largest positive integer $r$ such that $S^{r}$, the direct product of $r$ copies of $S$, can be generated by 2 elements. (The positive integer $\delta$ is well-defined. To see this, first note that it is known that every non-abelian finite simple group can be generated by 2 elements. Also, for any positive integer $d$, the group $S^{r}$ cannot be generated by $d$ elements whenever $r$ is larger than the number of $\operatorname{Aut}(S)$-orbits on the set of $d$-tuples generating $S$. This latter claim follows from the combination of the definition of a maximal subgroup of product type and the Pigeonhole Principle.) Let us denote $S^{\delta}$ by $G$. (Actually, $\delta$ is equal to the number of $\operatorname{Aut}(S)$-orbits on ordered pairs of generators for $S$, and for arbitrary elements $x=\left(x_{1}, \ldots, x_{\delta}\right)$ and $y=\left(y_{1}, \ldots, y_{\delta}\right)$ of $G$ we have that $G=\langle x, y\rangle$ if and only if the pairs $\left(x_{i}, y_{i}\right)$ are distinct representatives for these orbits for $i$ with $1 \leq i \leq \delta$.)

Consider $A=\operatorname{Aut}(G) \cong \operatorname{Aut}(S)<\operatorname{Sym}(\delta)$ and let $(x, y)$ be a fixed pair of generators for $G$ with $x=\left(x_{1}, \ldots, x_{\delta}\right)$ and $y=\left(y_{1}, \ldots, y_{\delta}\right)$, where the $x_{i}$ 's and $y_{i}$ 's are elements of $S$. Since $\langle x, y\rangle=G$, the elements $\left(x_{1}, y_{1}\right), \ldots,\left(x_{\delta}, y_{\delta}\right)$ form a set of representatives for the $\operatorname{Aut}(S)$-orbits of the set of generating pairs for $S$. From this it is easy to see that $G$ has the following relevant property: $(\mathcal{P})$ if $G=\langle\bar{x}, \bar{y}\rangle$, then there exists $a \in A$ with $(\bar{x}, \bar{y})=\left(x^{a}, y^{a}\right)$.

Now we can define a graph $\Gamma$ in which the set of vertices $V$ is the set of all $A$-conjugates of $x$ and two vertices $\bar{x}_{1}, \bar{x}_{2}$ are connected by an edge if and only if $G=\left\langle\bar{x}_{1}, \bar{x}_{2}\right\rangle$. Note that $\Gamma$ is obtained from $\Gamma(G)$ just by removing all isolated vertices. By property $(\mathcal{P})$, the graph $\Gamma$ is vertex-transitive and edge-transitive. Let $\alpha=|A|, C=C_{A}(x)$, and $\gamma=|C|$. The number of vertices in $V$ is $\alpha / \gamma$, and the number of edges in $\Gamma$ is $\alpha / 2$ (since the action of $A$ on the pairs of generators is regular and 2 in the denominator comes from the fact that the edges of $\Gamma$ are unoriented).

In the remainder of this section we wish to give an upper bound for $\omega(G)$ which is precisely the clique number of $\Gamma$.

We will use Corollary 4 of 4 which states that if $X$ is a clique and $Y$ a coclique (an empty subgraph) in a vertex-transitive graph on $m$ vertices, then $|X||Y| \leq m$.

We also need a definition. Let $s$ be an element of $S$ and let $\omega(s)$ be the number of indices $i$ with $1 \leq i \leq \delta$ such that $x_{i}$ and $s$ are $\operatorname{Aut}(S)$-conjugate. We have $\omega(s)=\rho(s) /\left|C_{\operatorname{Aut}(S)}(s)\right|$, where $\rho(s)$ is the number of elements $t$ in $S$ such that $\langle s, t\rangle=S$ (this is because for any $t$ with $\langle s, t\rangle=S$ there exists a unique index $i$ with $1 \leq i \leq \delta$ and a unique automorphism $a \in \operatorname{Aut}(S)$ such that $\left.\left(s^{a}, t^{a}\right)=\left(x_{i}, y_{i}\right)\right)$.

Now take $M$ to be a maximal subgroup of $S$ and put

$$
Y_{M}=\left\{v=\left(z_{1}, \ldots, z_{\delta}\right) \in V \mid \pi_{1}(v)=z_{1} \in M\right\}
$$

where $\pi_{1}$ is the natural projection from $G$ to the first direct factor. Since $Y_{M}$ is a coclique in $\Gamma$ we have $\omega(G) \leq|V| /\left|Y_{M}\right|$.

For any $z \in M$ with $z \neq 1$ there exists a vertex $v_{z}$ in $V$ such that $\pi_{1}\left(v_{z}\right)=z$. (This follows from the corollary on page 745 of [8], which states that any nontrivial element of a finite almost simple group $G$ belongs to a pair of elements 
generating at least the socle of $G$.) Other vertices $v$ with the property that $\pi_{1}(v)=z$ can be obtained by conjugating $v_{z}$ by automorphisms from the subgroup $\bar{A} \cong$ $\operatorname{Aut}(S)$ ? $\operatorname{Sym}(\delta-1)$ of $A$. So if we define $C_{z}$ to be $C_{\bar{A}}\left(v_{z}\right)$, then we obtain

$$
\sum_{z \in M, z \neq 1} \frac{|\bar{A}|}{\left|C_{z}\right|} \leq\left|Y_{M}\right|
$$

This implies

$$
\omega(G) \leq \frac{|V|}{\sum_{z \in M, z \neq 1} \frac{|\bar{A}|}{\left|C_{z}\right|}}=\left(\sum_{z \in M, z \neq 1} \frac{|\bar{A}||C|}{|A|\left|C_{z}\right|}\right)^{-1} .
$$

Clearly $|A| /|\bar{A}|=\delta|\operatorname{Aut}(S)|$. Now assume that $\left\{u_{1}, \ldots, u_{l}\right\}$ is a set of representatives for the orbits of the action of $\operatorname{Aut}(S)$ on $S \backslash\{1\}$. For $C=C_{A}(x)$ we have

$$
C \cong \prod_{i=1}^{l} C_{\operatorname{Aut}(S)}\left(u_{i}\right) \prec \operatorname{Sym}\left(\omega\left(u_{i}\right)\right) \text {. }
$$

On the other hand, if $z \in u_{j}^{\operatorname{Aut}(S)}$, we have

$$
C_{z} \cong\left(\prod_{i \neq j} C_{\operatorname{Aut}(S)}\left(u_{i}\right) \prec \operatorname{Sym}\left(\omega\left(u_{i}\right)\right)\right) \times\left(C_{\operatorname{Aut}(S)}\left(u_{i}\right) \prec \operatorname{Sym}\left(\omega\left(u_{i}\right)-1\right)\right) .
$$

It follows that $|C| /\left|C_{z}\right|=\left|C_{\operatorname{Aut}(S)}(z)\right| \cdot \omega(z)=\rho(z)$ and

$$
\omega(G) \leq\left(\sum_{z \in M, z \neq 1} \frac{\rho(z)}{|\operatorname{Aut}(S)| \delta}\right)^{-1} .
$$

Note that $|\operatorname{Aut}(S)| \delta$ is the number of ordered pairs $(s, t)$ generating $S$, while $\sum_{z \in M, z \neq 1} \rho(z)$ is the number of ordered pairs $(s, t)$ generating $S$ such that $s \in M$. So if we define $P_{M}$ to be the conditional probability that $(s, t) \in M \times S$ given that $\langle s, t\rangle=S$, then $\omega(G) \leq 1 / P_{M}$. We may also write $P_{M}$ in the form

$$
P_{M}=\frac{P(\langle x, y\rangle=S \mid x \in M) \cdot P(x \in M)}{P(\langle x, y\rangle=S)} \geq P(\langle x, y\rangle=S \mid x \in M) \cdot \frac{|M|}{|S|},
$$

where $Q_{M}=P(\langle x, y\rangle=S \mid x \in M)$ is the conditional probability that the ordered pair $(x, y)$ generates $S$ given that $x \in M$, where $P(x \in M)=|M| /|S|$ is the probability that $x \in M$ and where $P(\langle x, y\rangle=S)$ is the probability that the ordered pair $(x, y)$ generates $S$. Clearly, $\omega(G) \leq 1 / P_{M} \leq|S: M| / Q_{M}$. We need a lower bound for $Q_{M}$. In what follows $m(S)$ denotes the minimal index of a proper subgroup in $S$.

Proposition 3.2. Let $M \leq S$ with $|S: M|=m(S)$. Then $1-O\left(m(S)^{-1 / 15}\right) \leq$ $Q_{M}$. Moreover if $S=\operatorname{Alt}(n)$, then $1-O\left(n^{-1}\right) \leq Q_{M}$.

Proof. If $(m, s) \in M \times S$, then $\langle m, s\rangle \neq S$ if and only if $(m, s) \in(K \cap M) \times K$ for some maximal subgroup $K$ of $S$. This allows us to deduce

$$
1-\sum_{K} \frac{1}{|S: K||M: K \cap M|} \leq Q_{M},
$$

where $K$ runs through the set of maximal subgroups of $S$. 
Now use the notation of Section 6 of 9 . There exist positive real numbers $\delta$ and $b$ with $\delta>1$ such that the set $\mathcal{A}$ of maximal subgroups whose index is smaller than $b \cdot m(S)^{\delta}$ is known (and $|\mathcal{A}|$ is "small"). The values of $\delta$ and $b$ together with the description of $\mathcal{A}$ are given in [9] when $S$ is a simple group of Lie type. If $S=\operatorname{Alt}(n)$ and $n$ is large enough, then any subgroup of $\operatorname{Alt}(n)$ different from a point-stabilizer has index at least $n(n-1) / 2$, so for any $\delta$ with $1<\delta \leq 2$ there exists $b>0$ with the property that any maximal subgroup of $\operatorname{Alt}(n)$ with index smaller than $b \cdot n^{\delta}$ is a point-stabilizer. By [9], we may take $\delta=16 / 15$ if $S$ is a group of Lie type, and, by the remarks above, we may take $\delta=2$ if $S$ is an alternating group. Let $\mathcal{B}$ be the set of those maximal subgroups of $S$ which do not belong to $\mathcal{A}$. Note that

$$
\frac{1}{|S: K||M: K \cap M|} \leq \frac{m(S)}{|S: K|^{2}} \text {. }
$$

We will make use of the identity

$$
\sum_{K \in \mathcal{B}}|S: K|^{-2}=O\left(m(S)^{-\delta}\right)
$$

which, for exceptional groups $S$ of Lie type, is found in line -2 of the proof of Lemma 6.7 in [9, and which, for classical groups $S$, follows from Theorem 3.1 of [10] by noting that we may replace 2 by $\delta$ since $\delta \leq 2$. This implies

$$
\sum_{K \in \mathcal{B}}|S: K|^{-1}|M: K \cap M|^{-1}=O\left(m(S)^{-\delta+1}\right) .
$$

Hence

$$
1-\sum_{K \in \mathcal{A}} \frac{1}{|S: K \| M: K \cap M|}-O\left(m(S)^{-\delta+1}\right) \leq Q_{M}
$$

Now let $\left\{K_{1}, \ldots, K_{t}\right\}$ be a set of representatives for the $S$-conjugacy classes of all members of $\mathcal{A}$. For every $i$ with $1 \leq i \leq t$ let $s_{i}$ be the number of $M$-orbits on the coset space $\left(S: K_{i}\right)$. Note (see the proof of Lemma 6.10 in [9]) that for every $i$ with $1 \leq i \leq t$ we have

$$
\sum_{K \in K_{i}^{G}} \frac{1}{|S: K||M: K \cap M|}=\frac{s_{i}}{\left|S: K_{i}\right|} \leq \frac{s_{i}}{m(S)} .
$$

We conclude that

$$
1-\sum_{i=1}^{t} \frac{s_{i}}{m(S)}-O\left(m(S)^{-\delta+1}\right) \leq Q_{M} .
$$

We now have to show that $\sum_{i=1}^{t} s_{i}$ is "small". If $S$ is a group of Lie type, then, by [9], $t \leq 3$ and either $s_{i} \leq 3$ for all $i$ with $1 \leq i \leq t$ or $S=P \Omega_{2 m}^{ \pm}(q)$ in which case there exists a constant $c_{1}$ such that $s_{i} \leq c_{1} q$ for all $i$ with $1 \leq i \leq t$ (see the last part of the proof of Lemma 6.7 in [9]). Finally, if $S=\operatorname{Alt}(n)$ and $n \neq 6$, then $t=1$ and $s_{1}$ is the number of orbits of the point-stabilizer $M$ on the coset space $\left(S: K_{1}\right)$ where $K_{1}$ is another point-stabilizer. In this case $s_{1}=2$ since $\operatorname{Alt}(n)$ is 2 -transitive. By these remarks and by inequality (2), we get

$$
1-O\left(m(S)^{-\delta+1}\right) \leq Q_{M},
$$

which is exactly what we wanted. 
By the inequality $\omega(G) \leq|S: M| / Q_{M}$ and by Proposition 3.2, we conclude that $\omega(G) \leq m(S)+O\left(m(S)^{14 / 15}\right)$ if $S$ is a finite simple group of Lie type and $\omega(G) \leq m(S)+O(1)$ otherwise. Now let $S=\operatorname{Alt}(n)$. Then, by 12, we have $2^{n-2} \leq \sigma(S)$ unless $n=7$ or 9 . Hence, by Proposition 3.1, $2^{n-2} \leq \sigma(G)$ unless $n=7$ or 9 . From this it follows that $\omega(G) / \sigma(G) \leq(n+O(1)) / 2^{n-2}$. The proof of Theorem 1.2 is now complete.

\section{REFERENCES}

[1] Abdollahi, A.; Jafarian Amiri, S. M. Minimal coverings of completely reducible groups. Publ. Math. Debrecen 72/1-2 (2008), 167-172. MR2376867(2008k:20044)

[2] Aschbacher, M.; Guralnick, R. M. Some applications of the first cohomology group. J. Algebra 90 (1984), 446-460. MR760022 (86m:20060)

[3] Blackburn, S. Sets of permutations that generate the symmetric group pairwise. J. Combin. Theory Ser. A 113 (2006), no. 7, 1572-1581. MR2259081(2007e:20005)

[4] Cameron, P. J.; Ku, C. Y. Intersecting families of permutations. European J. Combin. 24 (2003), no. 7, 881-890. MR.2009400 (2004g:20003)

[5] Detomi, E.; Lucchini, A. Crowns and factorization of the probabilistic zeta function of a finite group. J. Algebra 265 (2003), no. 2, 651-668. MR1987022 (2004e:20119)

[6] Dye, R. H. Interrelations of symplectic and orthogonal groups in characteristic two. J. Algebra 59 (1979), no. 1, 202-221. MR541675 (81c:20028)

[7] Gaschütz, W. Praefrattinigruppen. Arch. Math. (Basel) 13 (1962), 418-426. MR0146262 $(26: 3784)$

[8] Guralnick, R. M.; Kantor, W. M. Probabilistic generation of finite simple groups. Special issue in honor of Helmut Wielandt. J. Algebra 234 (2000), no. 2, 743-792. MR.1800754 (2002f:20038)

[9] Liebeck, M. W.; Shalev, A. Simple groups, probabilistic methods, and a conjecture of Kantor and Lubotzky. J. Algebra 184 (1996), no. 1, 31-57. MR.1402569 (97e:20106b)

[10] Liebeck, M. W.; Shalev, A. Classical groups, probabilistic methods, and the (2,3)-generation problem. Ann. of Math. (2) 144 (1996), no. 1, 77-125. MR1405944 (97e:20106a)

[11] Lucchini, A.; Maróti, A. On finite simple groups and Kneser graphs, J. Algebraic Combin., to appear.

[12] Maróti, A. Covering the symmetric groups with proper subgroups. J. Combin. Theory Ser. A 110 (2005), no. 1, 97-111. MR2128968 (2005m:20009)

[13] Robinson, D. J. S. A course in the theory of groups, Graduate Texts in Mathematics, 80, Springer-Verlag, New York, 1993. MR 1261639 (94m:20001)

[14] Tomkinson, M. J. Groups as the union of proper subgroups. Math. Scand. 81 (1997), 191-198. MR:1613772 (99g:20042)

Dipartimento di Matematica Pura ed Applicata, Università di Padova, Via Trieste 63, 35121 Padova, ItALY

E-mail address: lucchini@math.unipd.it

Institute of Mathematics, Hungarian Academy of Sciences, Reáltanoda utca 13-15, H-1053, Budapest, Hungary

E-mail address: maroti@renyi.hu 